\title{
ORIGINAL
}

\section{RECOMENDACIONES PARA LA INVESTIGACIÓN E INFORMACIÓN EN SALUD SOBRE DEFINICIONES Y VARIABLES PARA EL ESTUDIO DE LA POBLACIÓN INMIGRANTE DE ORIGEN EXTRANJERO}

\author{
Davide Malmusi (1,2), Josep Maria Jansà i Lopez del Vallado (1).
}

(1) Agència de Salut Pública de Barcelona.

(2) UD Medicina Preventiva i Salut Pública IMAS-UPF-ASPB.

\section{RESUMEN}

Fundamento: el crecimiento de la población extranjera residente en el Estado español requiere disponer de mejor información sobre su nivel de salud. En los sistemas de información e investigación en salud no existe un criterio uniforme para la nomenclatura de las variables de origen. Este estudio plantea un consenso sobre las definiciones de inmigrante y extranjero.

Métodos: siguiendo la metodología Delphi se invitó a 66 expertos en inmigración de distintas disciplinas a participar en el estudio, utlizando un cuestionario en dos vueltas con propuestas de definiciones, términos y variables e incluyéndose en la segunda los porcentajes de respuestas y los comentarios de los participantes de la primera. Las propuestas con un acuerdo del $80 \%$ se trasladaron al documento final que fue aprobado en una tercera vuelta.

Resultados: respondieron 57 personas a la invitación, 44 completaron al menos una ronda y 33 completaron las tres. Entre los elementos consensuados destacan: se nombra inmigrante a la persona que llega a un país donde no ha nacido para fijar su residencia; no se es inmigrante de por vida. Puede considerarse inmigrante a las personas nacidas en otro país llegadas a España desde hace menos de 5-10 años; y personas inmigradas son todas las nacidas en otro país. Cabe además considerar variables socioeconómicas, género, situación administrativa y lugar de procedencia. Los hijos de personas inmigradas nacidos en España no deben considerarse inmigrantes.

Conclusiones: Para definir adecuadamente las categorías de población inmigrante e inmigrada los sistemas de información y estudios en salud deben recoger país de nacimiento, año de llegada y nacionalidad.

Palabras clave: Inmigración. Inmigrante. Método Delphi. Sistemas de información.

Correspondencia:

Davide Malmusi

Unitat Docent de Medicina Preventiva i Salut Pública.

Servei d'Avaluació i Epidemiologia Clínica

Hospital del Mar.

Passeig Marítim, 25-29. 08071 Barcelona

Correo electrónico: dmalmusi@aspb.es

\section{ABSTRACT}

\section{Recommendations for Health Research and Information on Definitions and Variables for the Study of the Foreign-Born Immigrant Population}

Background: The growth of the foreign population residing in Spain makes it necessary to avail of better information on the level of health. In the health information and research systems, no uniform criteria currently exist for the nomenclature of origin-related variables. This study poses a consensus on the definitions of immigrant and foreigner.

Methods: Employing the Delphi methodology, 66 experts in immigration from different disciplines were invited to take part in the study using a two-round questionnaire with suggested definitions, terms and variables, including in the second round the percentages of responses and the comments made by the participants in the first. The suggestions regarding which an $80 \%$ consensus was reached were put into a final document which was approved in the third round.

Results: A total of 57 people accepted the invitation, 44 having completed at least one round and 33 having completed all three. Some of the most important aspects regarding which a consensus was reached were: the term "immigrant" denotes the individual who comes to a country where he/she was not born to take up residency, one not being an immigrant on a lifelong basis. Those individuals born in another country who have arrived in Spain less than 5-10 years ago can be considered immigrants; and immigrated people are all those individuals born in another country. Socioeconomic variables, gender, administrative status and country of origin should also be taken into consideration. The children of immigrated individuals who are born in Spain must not be considered individuals who are born in Spain must not be considered.

Conclusions: To properly define the inmigrating and immigrated population categories, the health studies and information systems must include country of birth, year of arrival and nationality.

Keywords: Immigration. Migrant. Delphi technique. Information systems. Data collection. 


\section{INTRODUCCIÓN}

En los últimos años la inmigración extranjera en el estado español se ha situado en primera línea de los fenómenos sociodemográficos. Así, en enero de 2007 estaban empadronados en España 4.482.568 extranjeros, lo que representaba en torno al $10 \%$ de la población del país ${ }^{1}$. La cifra global de extranjeros se ha incrementado en más de tres millones desde el año 1999, cuando representaba sólo un $1,8 \%$ de la población total, configurándose rápidamente una nueva realidad social y demográfica en el conjunto del país.

Este cambio requiere, entre otros aspectos, disponer de información sobre la salud y principales necesidades sanitarias de esta población. En España los estudios dirigidos a analizar la salud de los inmigrantes se han incrementado en los últimos años, a pesar de que en su mayoría presentan importantes carencias metodológicas ${ }^{2}$. Distintas revisiones recomendaban la inclusión en los registros sanitarios de variables específicas que permitan analizar el patrón de morbilidad y mortalidad de la población inmigrante ${ }^{2-4}$. Una revisión de la situación en la ciudad de Barcelona puso de manifiesto que no todos los sistemas de información permiten identificar a los ciudadanos y usuarios de origen extranjero y que los que sí lo hacen no utilizan las mismas variables (nacionalidad, país de nacimiento, país de origen $)^{5}$. En este sentido, la obtención de datos sobre "la salud de los inmigrantes" requiere precisar a quien se hace referencia al utilizar este término.

La mayoría de fuentes estadísticas en España, empezando por el $\mathrm{INE}^{1}$, se centran en la categoría jurídica de extranjero (personas residentes en el territorio español sin nacionalidad española), lo que impide incluir a quiénes han adquirido la nacionalidad española o a sus hijos nacidos en nuestro país, cuando para estas poblaciones sería interesante identificar desigualdades sociales, de salud o en la utilización de recursos ${ }^{6}$. Por otro lado, según los diccionarios inmigrante es el que inmigra, donde inmigrar significa "llegar a otro país para establecerse en él"7, mientras que la imagen social y mediatizada de los inmigrantes acostumbra a restringirse a la de una persona "diferente" en rasgos somáticos y con problemas de marginalidad y pobreza ${ }^{6}$. En los estudios del sector sanitario es frecuente excluir de la definición de inmigrante a las personas originarias de países de la Unión Europea y/o de países desarrolla$\operatorname{dos}^{8}$.

A esta falta de uniformidad no son ajenos otros países de larga tradición migratoria, cuyas estadísticas públicas, si bien se centran en su mayoría en el país de nacimiento, utilizan términos y definiciones distintas, incluyendo o excluyendo de la población inmigrante por ejemplo a las personas nacidas en el país de padres originarios de otro?.

Dadas las limitaciones y la falta de acuerdo en la identificación de la población inmigrante $^{2,5}$ y para unificar criterios en los sistemas de información y en la investigación en salud, el presente trabajo se propuso lograr un consenso entre expertos de Cataluña sobre los términos, definiciones y variables más adecuados para caracterizar a la población de origen extranjero en los ámbitos de la salud y las ciencias sociales.

\section{SUJETOS Y MÉTODOS}

Para lograr el mencionado consenso se constituyó entre febrero y abril del 2006 un grupo de expertos según la metodología Delphi ${ }^{10,11}$. Esta técnica que nació y tuvo su mayor desarrollo como instrumento para predicciones de escenarios futuros y que se ha utilizado para valorar el acuerdo de un grupo respecto a determinadas afirmaciones $^{12,13}$, se basa en la interacción anónima de los participantes a través de 
rondas sucesivas, tal como se describe más adelante.

Selección de los participantes. Para crear un grupo con personas de referencia en los temas de inmigración en Cataluña (ámbito del estudio) se elaboró un listado a partir de distintas fuentes: miembros del consejo asesor del Pla Director d'Immigració del Departament de Salut de la Generalitat de Catalunya, miembros del grupo de inmigración del Consell Municipal de Benestar Social del Ayuntamiento de Barcelona, autores de bibliografía relevante en los últimos cinco años y profesorado de cursos y másteres sobre inmigración. Se intentó incluir una representación equilibrada de personas procedentes de los ámbitos de la salud y de otros entornos académicos y profesionales como sociología, antropología y derecho; administraciones públicas; asociaciones de inmigrados o dedicadas al trabajo con esta población inmigrante. Considerando una tasa mínima de respuesta de un $50 \%$, a pesar de que en la mayoría de los casos las tasas sean más elevadas ${ }^{12,13}$, y con la intención de superar la cuota mínima aconsejada de 25-30 sujetos $^{11}$, se invitó finalmente a participar a 66 personas.

Para averiguar la existencia de agrupaciones entre las pérdidas en el proceso, se comparó la composición del grupo a lo largo de las rondas según lugar de nacimiento, nacionalidad y formación inicial. Entre las personas con formación en ciencias sociales también se distinguió si su ocupación actual se vinculaba a temas de salud. Asimismo, los profesionales sanitarios se agruparon de acuerdo a si su ocupación era en la clínica o en salud pública. Para cada subgrupo se calculó la tasa de abandono como la proporción de personas que no llegaron hasta la última ronda sobre el total de personas contactadas.

Preparación del cuestionario inicial. Se elaboró un cuestionario dividido por temas:
— Definición del término "inmigrante" y categorizaciones más relevantes para el estudio de esta población en el ámbito de la salud y de las ciencias sociales

- Términos más adecuados para denominar a las personas extranjeras

- Variables que deberían recogerse para definir a la población inmigrante

- Términos y definiciones para los hijos de inmigrantes que nacen en el país de destino

En cada apartado se planteaban distintas opciones de términos o definiciones a utilizar, sobre las cuales los participantes debían expresar su acuerdo o desacuerdo. Las opciones incluidas se inspiraban básicamente en la terminología y definiciones utilizadas en las instituciones oficiales de distintos países ${ }^{9}$, en la literatura científica y en los medios de comunicación.

El borrador de cuestionario fue discutido con dos personas que habían utilizado en otras ocasiones el método Delphi para el establecimiento de consenso, y enviado a 12 personas seleccionadas para participar en la prueba piloto, para que lo respondieran y aportaran sus comentarios sobre la comprensibilidad y adecuación de las preguntas.

Desarrollo del consenso. El cuestionario se envió por correo electrónico, dándose en cada ronda 15 días para la respuesta. Para cada opción del cuestionario (p.ej. propuesta de definición o término a utilizar) era posible contestar según 4 categorías: 'totalmente de acuerdo', 'de acuerdo', 'en desacuerdo', 'totalmente en desacuerdo’. Además era posible redactar comentarios o proponer nuevas opciones de respuesta. Se fijó el $80 \%$ como cuota para la aprobación o rechazo de cada opción (aceptación de opciones con un 80\% de "totalmente de acuerdo o de acuerdo" $\mathrm{y}$ 
rechazo de aquéllas con el $80 \%$ de "desacuerdo o totalmente en desacuerdo").

Las respuestas recibidas se elaboraron y reenviaron a los participantes en forma de porcentajes para cada propuesta según categorías, juntamente con los comentarios expresados en forma anónima. Conociendo las primeras respuestas y comentarios del grupo, la segunda ronda consistió en volver a contestar a las opciones sin consenso y a las nuevas opciones y preguntas surgidas. A raíz de los resultados de las dos rondas se preparó un documento de consenso que incorporaba las afirmaciones que el grupo había aceptado o rechazado por separado, e intentaba resolver puntos sin consenso a través de nuevas formulaciones de compromiso. Para la tercera ronda se pidió a los participantes que marcaran las partes, frases y palabras del documento con las cuales no estaban de acuerdo, siempre con la posibilidad de formular comentarios y propuestas alternativas. El criterio del $80 \%$ de acuerdo se extendió en este caso a las frases enteras, eliminando aquellas sin consenso e incorporando pequeñas propuestas de mejora formal, con lo que se llegó al documento final.

\section{RESULTADOS}

\section{Características de los participantes}

De las 66 personas invitadas a participar no se pudo localizar a 7 y 2 contestaron cuando ya se había iniciado el proceso. De las 57 personas contactadas a tiempo 4 rechazaron participar y 9 de las que en un principio habían aceptado finalmente no llegaron a contestar a ninguna ronda. Finalmente, 44 expertos (el 66,7\% de los inicialmente invitados) respondieron a la primera ronda, 37 a la segunda y 33 (el $75 \%$ de los que habían contestado a la primera y el $50 \%$ de los inicialmente invitados) a la tercera y última ronda.

En la tabla 1 se muestra la composición del grupo según lugar de nacimiento, nacio-

\section{Tabla 1}

Estudio Delphi: Características de los participantes

\begin{tabular}{|c|c|c|c|c|}
\hline & Contactados & Primera ronda & Última ronda & Tasa de abandono* \\
\hline & $\mathrm{N}(\%)$ & $\mathrm{N}(\%)$ & $\mathrm{N}(\%)$ & $\%$ \\
\hline & 57 & 44 & 33 & 42 \\
\hline $\begin{array}{r}\text { Lugar de nacimiento } \\
\text { Cataluña } \\
\text { Resto de España } \\
\text { Extranjero }\end{array}$ & $\begin{array}{r}33(58) \\
9(16) \\
15(26)\end{array}$ & $\begin{array}{r}25(57) \\
6(14) \\
13(30)\end{array}$ & $\begin{array}{r}17(52) \\
4(12) \\
12(36)\end{array}$ & $\begin{array}{l}48,5 \\
55,6 \\
20,0\end{array}$ \\
\hline $\begin{array}{r}\text { Española } \\
\text { Extranjera } \\
\text { Ambas }\end{array}$ & $\begin{array}{r}46(81) \\
8(14) \\
3 \quad(5)\end{array}$ & $\begin{array}{r}34(77) \\
7(16) \\
3 \quad(7)\end{array}$ & $\begin{array}{c}24(73) \\
7(21) \\
2,0(6)\end{array}$ & $\begin{array}{l}47,8 \\
12,5 \\
33,3\end{array}$ \\
\hline $\begin{array}{l}\text { Área de formación } \\
\text { Ciencias de la salud } \\
\begin{array}{rr}\text { Medicina clínica } \\
\text { Salud pública }\end{array} \\
\begin{array}{rr}\text { Ciencias sociales } \quad \begin{array}{l}\text { Derecho } \\
\text { Sociología }\end{array} \\
\text { Relación con el ámbito de salud }\end{array}\end{array}$ & $\begin{array}{r}14(25) \\
7(12) \\
7(12) \\
42(75) \\
6(11) \\
9(16) \\
10(18)\end{array}$ & $\begin{array}{r}10(23) \\
3(7) \\
7(16) \\
34(77) \\
5(11) \\
8(18) \\
10(23)\end{array}$ & $\begin{array}{r}8(24) \\
1(3) \\
7(21) \\
25(76) \\
1(3) \\
6(18) \\
10(30)\end{array}$ & $\begin{array}{c}42,9 \\
85,7 \\
0,0 \\
40,5 \\
83,3 \\
33,3 \\
0,0\end{array}$ \\
\hline $\begin{array}{l}\text { Área de trabajo } \\
\text { Universitarios / investigadores } \\
\text { Administración pública } \\
\text { Otras entidades }\end{array}$ & $\begin{array}{l}32(56) \\
11(19) \\
14(25)\end{array}$ & $\begin{array}{r}24(55) \\
9(20) \\
11(25)\end{array}$ & $\begin{array}{r}19(58) \\
7(21) \\
7(21)\end{array}$ & $\begin{array}{l}40,6 \\
36,4 \\
50,0\end{array}$ \\
\hline
\end{tabular}

* [(Contactados - Participantes en la última ronda) / Contactados $] * 100$. 
nalidad, formación y área de trabajo. El 26\% de las personas contactadas habían nacido en el extranjero y el $28 \%$ eran profesionales del ámbito de las ciencias de la salud. El rechazo o abandono fue más frecuente entre las personas nacidas en España y entre el personal clínico y los juristas, frente a los profesionales de salud pública y las personas formadas en ciencias sociales que trabajan en relación con el ámbito de salud.

\section{Rondas de consenso}

Tal como se ha descrito en la metodología, se desarrollaron tres rondas durante las cuales los participantes opinaron sucesivamente sobre un primer cuestionario, una versión modificada y un borrador de documento final de consenso. A lo largo de las 3 rondas, los 44 participantes realizaron 239 comentarios distintos, con más de 11.000 palabras, lo que equivale a 5,5 comentarios y 250 palabras por participante. El documento final se muestra en el anexo 1. Seguidamente se sintetiza el desarrollo del debate según apartados.

Definición del termino "inmigrante". De 16 propuestas de definición presentadas sólo una obtuvo más del $80 \%$ de acuerdo: "persona que llega a un país dónde no ha nacido para fijar su residencia". El grado de acuerdo para esta definición fue elevado en todos los grupos de origen, formación y ámbito de trabajo. Las demás propuestas, menos una muy similar a la aceptada, sólo alcanzaron el $60 \%$ de acuerdo o menos, agrupándose de acuerdo a si:

- suponían que era "inmigrante" toda persona que vivía en un país procedente de otro distinto. Algunos veían en el término un componente de transición, de proceso en curso, por lo que en la segunda ronda el $80 \%$ del grupo rechazó el enunciado: "Las personas llegadas de otro país siguen siendo inmigrantes, aún cuando lleven muchos años".
- incluían a los hijos nacidos en España de padres nacidos en otro país; como se explica en el último apartado de resultados, en el documento final se aprobó que "ya no deberían considerarse inmigrantes" (anexo 1).

- suponían que eran inmigrantes sólo las personas procedentes de países pobres; idea rechazada por el grupo (88\%).

Definición operativa. Se empezó por plantear cual era la mejor variable para definir quien es inmigrante entre el país de nacimiento y la nacionalidad. El 55\% de los participantes se decantaron por la primera, el $36 \%$ por la segunda, y los demás hicieron otras propuestas. En la segunda ronda el 69\% aprobó la combinación de ambas variables: haber nacido en el extranjero y no disponer de nacionalidad española. De los comentarios se deduce, por un lado, que quien prefiere la nacionalidad considera su adquisición como un paso fundamental en el proceso de asentamiento, y puede decirse que se deja no sólo de ser extranjero sino también inmigrante; mientras que hay quien considera este parámetro poco fiable, valorando que existen distintos requerimientos para la nacionalización, y defiende el país de nacimiento como variable que permite seguir en el tiempo a toda la población "inmigrada", y descartar a los hijos de padres extranjeros nacidos en España. La nacionalidad fue más respaldada por los miembros de la administración y los juristas, mientras que médicos, universitarios o investigadores, sociólogos y los participantes nacidos en otro país se inclinaron por el país de nacimiento.

No fue posible consensuar una definición operativa de país de origen, entendiéndolo como el lugar que más condiciona al individuo en términos de nacer, crecer, educarse, socializarse. Tampoco hubo consenso sobre indicadores de tiempo de asentamiento para su inclusión en la definición operativa de inmigrante. Se descartaron los años 
desde el empadronamiento o desde la consecución de un permiso de residencia, o el hecho de conseguir un permiso permanente; el $48 \%$ aprobó el uso del tiempo desde la llegada a España, siendo los puntos de corte propuestos de 5 ó 10 años, sin que se llegara al consenso sobre ninguno de los dos.

A partir de esta situación sin consenso se propuso considerar inmigrantes a las personas nacidas fuera de España "hasta un tiempo determinado desde su llegada, que, dependiendo del interés o finalidad del estudio, se puede fijar en 5 ó 10 años." (anexo 1).

Así mismo se asumió que cuando no estuviera disponible el año de llegada, como es el caso de las estadísticas actuales, se contabilizarían como inmigrantes a las personas nacidas fuera de España que siguen teniendo nacionalidad extranjera.

Por otro lado, si los inmigrantes son personas que dejan de tener esta condición al cabo de unos años, para estudiar a toda la población inmigrada de origen extranjero se aceptó incluir a todas las personas residentes en España nacidas en otro país (anexo 1).

Para aplicar estas definiciones, el grupo recomienda que los sistemas de información y protocolos de investigación recojan lugar de nacimiento, la nacionalidad y año de llegada a España. La primera sería la única variable indispensable para designar a la población inmigrada, aunque las otras dos serían fundamentales cuando se quiera restringir el análisis a los recién llegados o estudiar la relevancia del grado de asentamiento de una persona. Las definiciones consensuadas de personas inmigrantes e inmigradas y las variables necesarias para aplicarlas se resumen en la tabla 2.

Distinciones entre inmigrantes. En la primera ronda se preguntó si se consideraba legítima la distinción entre inmigrantes de países ricos o países pobres. El 80\% aprobó el uso de la distinción en el estudio de los problemas y perfiles sociales y de salud, aunque lo aceptaron sólo el 50\% de los sociólogos frente a todos los médicos y juristas, y se comentó la necesidad de ser cautos en su uso, sin por ejemplo atribuir a la condición de origen demasiadas consecuencias, pasando por alto las condiciones de vida en el lugar de destino. De hecho, se aprobó con un amplio margen que se tuvieran en cuenta otras características. Así, en la última ronda se pidió puntuar de 1 a 10 cada una de las características aprobadas según la importancia que se le otorgaba, la media de las puntuaciones dio lugar al orden en el cual estos factores se presentan en el documento final. El nivel de renta del país de nacimiento se consideró, en prácticamente

Tabla 2

Definiciones y variables para la población de origen extranjero

\begin{tabular}{|c|c|c|c|}
\hline \multirow{2}{*}{ Termino } & \multicolumn{2}{|c|}{ Definición } & \multirow{2}{*}{ Variables necesarias } \\
\hline & Conceptual & Operativa & \\
\hline \multirow[t]{2}{*}{ Inmigrante } & $\begin{array}{l}\text { Persona que llega a un país dónde } \\
\text { no ha nacido para fijar su residencia }\end{array}$ & $\begin{array}{l}\text { 1. personas nacidas fuera de } \\
\text { España y que han llegado desde } \\
\text { hace menos de } 5 \text { ó } 10^{*} \text { años }\end{array}$ & $\begin{array}{l}\text { Lugar de nacimiento; año de llega- } \\
\text { da; domicilio** }\end{array}$ \\
\hline & & $\begin{array}{l}\text { 2. personas nacidas fuera de Espa- } \\
\text { ña y de nacionalidad extranjera }\end{array}$ & $\begin{array}{l}\text { Lugar de nacimiento; nacionali- } \\
\text { dad; domicilio** }\end{array}$ \\
\hline Personas inmigradas & $\begin{array}{l}\text { Todas las que han llegado de otro } \\
\text { país }\end{array}$ & Personas nacidas fuera de España & Lugar de nacimiento; domicilio** \\
\hline
\end{tabular}

* El grupo no ha obtenido un consenso sobre este punto. Se sugiere valorar caso a caso dependiendo del objetivo del estudio y de la población que se quiere identificar.

** Necesario para distinguir de los turistas. 
todos los subgrupos, menos importante que la situación socioeconómica del individuo, el género, la regularización administrativa y la región geográfica.

El debate sobre el uso de etnia y rasgos físicos. Se planteó considerar como determinante el aspecto físico clasificado como "occidental / no occidental"; con el fin de captar el efecto de la discriminación en quienes a simple vista aparecen como diferentes, y procedentes de países de baja renta. Por la misma razón en el apartado relativo a los hijos de inmigrantes inicialmente se propuso seguir a las "minorías visibles" como posible objeto de desigualdades, término que en Canadá se utiliza en el censo y es objeto de políticas de discriminación positiva ${ }^{14}$. Ambas propuestas fueron rechazadas por el grupo. En algunos comentarios existía acuerdo con la idea, pero no con los términos; en otros se rechazaba por el efecto estigmatizador de los conceptos y por su sin-sentido en el caso de las personas adoptadas. La propuesta de tener en cuenta raza o etnia como características determinantes tampoco fue bien recibida (73\% de rechazo), así como el uso del término "minorías étnicas". Quedaba claro que no deben confundirse los conceptos de inmigrante y de minoría étnica (cuando es frecuente en la literatura el uso del país de nacimiento para definir etnias ${ }^{15,16}$ ), y que el termino puede conllevar la idea de marginación e la imposición de etiquetas.

Términos adecuados. Este apartado fue discutido por el aparente objetivo de poner orden entre términos "políticamente" correctos o incorrectos, y llevó a pocos consensos. Destacó que el $60 \%$ de los participantes manifestó desacuerdo con el uso de la expresión "inmigrantes económicos" para hacer referencia a quienes proceden de países pobres. El $78 \%$ estuvo de acuerdo con la denominación "inmigrantes de países de renta alta (IPRA) e inmigrantes de países de renta media ó baja (IPRB)". El 98\% rechazó el uso de la expresión "inmi- grantes ilegales" para aquellas personas que no disponen de permiso de residencia.

Términos y definiciones para los hijos de inmigrantes. En el documento final (anexo 1) se aprobó que no deberían considerarse inmigrantes, evitándose términos como "inmigrantes de segunda generación". Algunos participantes matizaron que se trata en todo caso de una población con características propias respecto a los hijos de autóctonos, sin embargo no se pactó una definición de quiénes deberían estudiarse como producto de la inmigración y posible objeto de desigualdades, debido al rechazo de una parte del grupo a crear esta distinción.

\section{DISCUSIÓN}

El consenso entre personas con experiencias personales y laborales muy diversas se obtiene de la consideración de diversos puntos de vista y situaciones, sin embargo, el abandono de algunos participantes en el estudio determinó que en la segunda ronda el grupo estuviera compuesto en un $45 \%$ por médicos o por personas con formación en ciencias sociales pero trabajando en temas de salud, lo que podría haber centrado la mayoría de las respuestas a situaciones de este ámbito.

Por otro lado, el producto del consenso podría considerarse más ideológico que científico. De algún modo este aspecto resulta inherente al tema del estudio ya que, a modo de ejemplo, no puede existir una definición científicamente probada de lo que es un inmigrante. En buena medida se hace referencia a un constructo social, cuyo significado está fuertemente influenciado por el contexto histórico. De hecho, el método Delphi y las técnicas de consenso son recursos que se utilizan ante la falta de ciencia cierta, y en general tienden a sacar a la luz la opinión dominante del momento sobre el tema tratado. Lo que sí se ha pre- 
tendido garantizar es la selección, a partir de fuentes objetivas, de una muestra de participantes con visiones de la realidad migratoria representativas de la mayoría de sectores expertos.

El papel y la influencia de los coordinadores del estudio constituye otro punto clave, ya que las preguntas y las opciones planteadas al principio, y las decisiones sobre los cambios en el cuestionario para avanzar en el debate y llegar a conclusiones pueden estar influidos por su visión y prioridades. Ciertamente se ha priorizado, por el interés en obtener recomendaciones para los sistemas de información y la investigación en salud, la obtención de definiciones operativas y recomendaciones claras sobre variables. Por lo demás, y para evitar dar ventaja a la visión de quien coordinó el trabajo, las respuestas y comentarios de los participantes se publicaron en su totalidad, y la introducción de nuevas preguntas siempre se justificó sobre la base de los resultados obtenidos y de las solicitudes recibidas. La satisfacción directamente expresada por algunos de los participantes sobre el desarrollo y gestión del debate apuntaría hacia la consecución de este objetivo.

Otro indicador claro de una buena participación es la cantidad de comentarios recibidos. Un incentivo para la participación viene dado por el método, ya que el anonimato en las respuestas y comentarios evita dinámicas de liderazgo, influencia o recelo propias de un grupo presencial, permitiendo que un participante pueda defender sus argumentos y cambiar sus opiniones con tranquilidad sin la influencia de otros miembros del grupo ${ }^{10,11}$.

Para avanzar en cuestiones complejas que quedaron sin consenso, tal vez hubiera sido beneficiosa una reunión presencial final, sin embargo esta opción se desestimó teniendo en cuenta el descenso en la participación entre la primera y la tercera ronda, tanto en el número de participantes como en la cantidad de comentarios. En este sentido, probablemente el consenso resultante de la reunión no hubiera sido ya representativo de la opinión del grupo, sino de la de sus componentes más motivados.

¿Tienen entonces los resultados validez interna y potencia? La impresión global refuerza esta tesis dado que el dintel requerido para el consenso $(80 \%)$ era similar al de otros estudios ${ }^{12,13}$, y en este caso el tema era muy indefinido y el grupo muy heterogéneo. El grado de participación indica que en general no hubo condescendencia en la aceptación de las propuestas. El análisis de las respuestas muestra una concordancia aceptable entre grupos de participantes de distinta procedencia geográfica y profesional. Si muchas frases del documento final pueden parecer obvias y de sentido común, hay que considerar el proceso de debate y "negociación" a partir del que se han obtenido, midiéndose cada palabra, con lo que su interpretación debe ser muy estricta.

¿A qué ámbitos pueden generalizarse y aplicarse? El primer objetivo era tener una base válida de consenso para elaborar recomendaciones en el ámbito de la información e investigación en salud en Cataluña. A pesar de esto, el estudio se ha diseñado para que sus resultados fueran aplicables al conjunto de las ciencias sociales. En este sentido, muchos participantes reconocieron la falta en sus contextos de trabajo, de un acuerdo claro, lo que hace pensar que las conclusiones del estudio puedan ser utilizadas en otras disciplinas. Respecto al ámbito geográfico a pesar de realizarse en Cataluña, y que el catalán fue la primera lengua de redacción del cuestionario y del documento conclusivo, la traducción del documento al castellano fue también aprobada por el grupo. Así mismo, la realidad de la inmigración en el conjunto del Estado presenta suficientes elementos comunes como para que permita a nuestro entender la utilización de los conceptos consensuados. La extrapolación a otros países europeos 
podría resultar más compleja, aunque a falta de otros estándares, y teniendo en cuenta las diferencias de contexto, estas conclusiones pueden constituir un nuevo elemento de referencia.

¿Son relevantes los resultados obtenidos? ¿Es importante para las ciencias sociales y de la salud disponer de un consenso de expertos sobre la terminología correcta, cuando seguramente las prioridades de los recién llegados son otras? Consideramos que no se trata de consensuar aquello que resulta "políticamente correcto", sino de aplicar el mayor rigor conceptual posible. Para avanzar en el conocimiento, es fundamental utilizar las mismas definiciones y categorías, como medida facilitadora de la comparabilidad entre estudios y para llegar a construir un modelo teórico coherente que impute los problemas a sus verdaderas causas.

$¿$ Cuales son las principales implicaciones de este estudio? Como mensaje clave para quien aborda el estudio de la población extranjera en la información e investigación sanitaria, es fundamental reconocer que la definición de inmigrante no es neutra; debiendo especificarse siempre a quien se hace referencia con el término utilizado y evitando asumir que inmigrante y extranjero son sinónimos. Ello determina la necesidad de registrar el lugar de nacimiento además de la nacionalidad. Tampoco puede considerarse inmigrante sólo a aquella persona que procede de un contexto de pobreza, dado que la decisión de migrar se relaciona con múltiples factores. El adjetivo "inmigradas" puede resultar más adecuado para referirse a un conjunto de personas con distintos niveles de asentamiento, que tienen en común el haber nacido en un país distinto de aquel en el cual residen. Dicha definición puede llevar a "malclasificar" individuos hijos de españoles que por un hecho puntual nacieron en el extranjero; sin embargo, se trata de casos poco frecuentes que no deberían afectar estimaciones váli- das para el conjunto de la población. También se debe ser cuidadoso a la hora de hacer agrupaciones, que tampoco son neutras, y deberían responder a un modelo teórico sobre la causalidad de los fenómenos estudiados. El año de la llegada, la condición socioeconómica y otros factores tienen que estar registrados para no detenerse en las agrupaciones según el país de origen.

Al finalizar este trabajo quedan diversas cuestiones abiertas que pueden constituir otras tantas líneas de investigación: por ejemplo, la validez y relevancia de distintos indicadores de asentamiento, como el tiempo de residencia, o el concepto de "origen autopercibido" (de donde eres? de donde te sientes?), la relación entre diferencias visibles y discriminación ${ }^{17}$, como hablar de estas diferencias sin estigmatizar ${ }^{18}$ y los perfiles y características propias de los hijos de inmigrantes nacidos en nuestro país. La investigación en salud puede contribuir al conocimiento de en que medida estos factores influyen en la salud de las personas.

Finalmente, la experiencia de este estudio nos lleva a afirmar que en un ámbito todavía novedoso en este país pero en el cual muchas personas están trabajando, es importante impulsar iniciativas de diálogo, debate y colaboración entre disciplinas, y entre grupos dentro de las mismas, para unir esfuerzos en la comprensión de las necesidades de las personas inmigradas.

\section{AGRADECIMIENTOS}

En el estudio Delphi realizado participaron las personas que figuran a continuación, a las cuales los autores agradecen su colaboración.

Emily Ahonen, Estanislao Alonso, Xavier Alonso, Marcelo Amable, Teresa Añé, Francesc Auquer, Silvina Berra, Anna Cabot, Marcel Cano, Marta Comas, Francesc Cots, Gonzalo Escobar, Xavier Giró, 
Elisabet González, Kàtia Lurbe, Juana Martín, Laura Mascarella, Elvira Méndez, Francisco Javier Moreno, Pau Mota, Mónica Nadal, Abdou Mawa Ndiaye, Andreu Olesti, Jesús Edison Ospina, Oscar Pascual, Victoria Porthé, Rosa Puigpinòs, Adil Qureshi, Luis Rajmil, Lluis Recolons, Carme Roca, Dan Rodriguez, Marc Sáez, Ghassan Saliba, Ramon Sanahuja, Elena Sánchez, Imma Sau, Vicky Serra-Sutton, Carlota Solé, Neus Torbisco y Josep Vilarmau.

\section{BIBLIOGRAFÍA}

1. Instituto Nacional de Estadística. Avance de padrón a 1 de enero de 2007. Datos provisionales. Disponible en: www.ine.es/inebase/ En: Demografía y población > Padrón municipal: explotación estadística y nomenclátor.

2. Berra S, Elorza Ricart JM, Bartomeu N, Hausmann S, Serra-Sutton V, Rajmil L. Necessitats en salut i utilització dels serveis sanitaris en la població immigrant a Catalunya. Revisió exhaustiva de la literatura científica. Barcelona: Agència d'Avaluació de Tecnologia i Recerca Mèdiques. CatSalut. Departament de Sanitat i Seguretat Social. Generalitat de Catalunya; 2004.

3. Jansà JM, Borrell C. Inmigración, desigualdades y atención primaria: situación actual y prioridades. Aten Primaria. 2002; 29: 466-8.

4. Grupo de trabajo en salud internacional. Consideraciones y recomendaciones en inmigración y salud. Madrid: Consejo Asesor del Ministerio de Sanidad y Consumo; 2003. Documento interno.

5. Puigpinós R, Borrell C. Sistemas de información e inmigración: entre la realidad y la utopía. Ponencia de la XVI Escuela de verano de Salud Pública; 19 de sep. 2005: Llatzaret de Maó.

6. Parella Rubio S. La població estrangera a Catalunya. En: Larios MJ, Nadal M. La immigració a Catalunya avui. Anuari 2004. Barcelona: Editorial Mediterrània, 2005.

7. Real Academia Española. Diccionario de la Lengua Española - Vigésima segunda edición [citado 10 de jun. 2007]. http://buscon.rae.es/draeI
8. Salazar A, Navarro-Calderon E, Abad I, Alberola V, Almela F, Borrás R et al. Diagnósticos al alta hospitalaria de las personas inmigrantes en la ciudad de Valencia (2001-2002). Rev Esp Salud Publica. 2003; 77: 713-723.

9. Migration Policy Group. Diversity Policies: Terminology and Monitoring [citado 26 de ene. 2006]. www.migpolgroup.com/diversity/infopages/2783.html

10. Jones J, Hunter D. Consensus methods for medical and health services research. BMJ. 1995; 311: 376-80.

11. Astigarraga E. El método Delphi [citado 10 de nov. 2006]. www.codesyntax.com/prospectiva/Metodo_delphi.pdf

12. Herdman M, Rajmil L, Ravens-Sieberer U, Bullinger M, Power M, Alonso J and the European Kidscreen and Disabkids groups. Expert consensus in the development of a European healthrelated quality of life measure for children and adolescents: a Delphi study. Acta Pediatr. 2002; 91: 1385-90.

13. Caminal J, Mundet X, Ponsà JA, Sánchez E, Casanova C. Las hospitalizaciones por ambulatory care sensitive conditions: selección del listado de códigos de diagnóstico válidos para España. Gac Sanit. 2001; 15: 128-141.

14. Department of Justice Canada. E-5.401, Employment Equity Act. Canada Gazette núm 18, $15 / 12 / 1995$.

15. Stronks K, Ravelli AC, Reijneveld SA. Immigrants in the Netherlands: equal access for equal needs? J Epidemiol Community Health. 2001; 55: 701-707.

16. Norredam M, Krasnik A, Moller ST, Keiding N, Joost MJ, Sonne NA. Emergency room utilization in Copenhagen: a comparison of immigrant groups and Danish-born residents. Scand J Public Health. 2004; 32: 53-59.

17. Krieger N. Discrimination and health. En: Berkman LF, Kawachi I, editores. Social Epidemiology. New York: Oxford University Press, 2000. p. 36-75.

18. Bhopal R. Is research into ethnicity and health racist, unsound or important science? BMJ. 1997; 314: 1751. 


\section{Anexo 1}

\section{Documento de consenso final}

\section{Grupo Delphi para el consenso sobre definiciones y variables a utilizar para caracterizar la población de origen extranjero en el ámbito de la salud y de las ciencias sociales}

\section{Documento final de conclusiones}

Definición de inmigrante. Se denomina inmigrante a la persona que llega a un país dónde no ha nacido para fijar su residencia.

La definición así redactada hace referencia a los inmigrantes internacionales (de un Estado al otro). La intención de fijar la residencia puede ser tanto temporal como permanente y no implica disponer de un permiso de residencia. No sólo quien procede de un país pobre es un inmigrante.

Consideraciones terminológicas. Los hijos nacidos en el Estado español de padres nacidos en otro país ya no se deberían considerar inmigrantes, ni, por lo tanto, denominar "inmigrantes de segunda generación" o personas "de origen inmigrante". Es mejor hablar de "hijos e hijas de inmigrantes" o de "hijos e hijas de familias inmigradas", recordando que, aun cuando puedan no disponer todavía de la nacionalidad española, deben considerarse españoles y catalanes a todos los efectos.

Las personas llegadas de otro país no siguen siendo "inmigrantes" de por vida. Por lo tanto, sería mejor hablar de personas "inmigradas" cuando se hace referencia a personas ya asentadas en nuestro país. Ninguno de estos términos debe servir, en todo caso, para etiquetar como "diferente", sino para distinguir en determinadas circunstancias, como estudiar problemáticas y desigualdades que les pueden afectar negativamente.

Definiciones operativas. En el ámbito de la investigación y para el análisis de datos específicos, se recomienda para uniformar criterios, utilizar una definición operativa que considere a las personas nacidas fuera del Estado español como inmigrantes hasta un tiempo determinado desde su llegada, que, dependiendo del interés o finalidad del estudio, se puede fijar en 5 ó 10 años (el grupo no consiguió consenso sobre este punto). $\mathrm{Si}$ el año de llegada no se encuentra disponible, se pueden considerar como inmigrantes las personas nacidas fuera de España con nacionalidad extranjera o, si sólo se hace referencia a población adulta, todas las personas con nacionalidad extranjera.
Cuando el interés se centra en todas las personas "inmigradas", se pueden considerar como tal todas las que viven en España y han nacido en otro país; siempre teniendo en cuenta la nacionalidad y el tiempo transcurrido desde la llegada como variables importantes para determinar el grado de asentamiento.

Variables necesarias. Por lo tanto, para definir y caracterizar a la población inmigrante y/o inmigrada, hace falta que los registros, sistemas de información y protocolos de investigación recojan el lugar de nacimiento de las personas, la nacionalidad y el año de llegada a España.

A la hora de estudiar los perfiles y problemas (de salud, sociales, etc.) de los inmigrantes, ante una realidad tan compleja y heterogénea, también es importante hacer distinciones según varias características, como son, en orden de importancia:

- Variables socioeconómicas (clase social, ocupación, nivel de renta...);

- Género / sexo;

- Disponer de una situación administrativa regularizada;

- Continente, región geográfica o país de procedencia (suponiendo que cuanto más sea posible diferenciar mejor, pero que a veces es necesario agrupar);

- Ser originario de un país de renta alta o de renta mediana / baja;

- Tener o no una nacionalidad de la Europa comunitaria;

- Nivel de estudios;

- Procedencia rural o urbana;

- Estatus de refugiado.

Finalmente, se rechaza que los inmigrantes sin permiso de residencia se denominen "ilegales", "irregulares" o en "situación ilegal", mientras que se puede hablar de inmigrantes "en situación irregular"; y se rechaza que tanto los inmigrantes como sus hijos se clasifiquen según tengan o no aspecto o rasgos físicos "occidentales". 